\title{
Automated Module for Product Identification by their Visual Characteristics
}

\author{
Sergiy Novoselov \\ ORCID 0000-0002-3190-0592 \\ Department of Computer-Integrated \\ Technologies, Automation and \\ Mechatronics \\ Kharkiv National University \\ of Radio Electronics \\ Kharkiv, Ukraine \\ sergiy.novoselov@nure.ua
}

\author{
Oksana Sychova \\ ORCID 0000-0002-0651-557X \\ Department of Computer-Integrated \\ Technologies, Automation and \\ Mechatronics \\ Kharkiv National University \\ of Radio Electronics \\ Kharkiv, Ukraine \\ sergiy.novoselov@nure.ua
}

\author{
Yevhenii Pashchenko \\ Department of Computer-Integrated \\ Technologies, Automation and \\ Mechatronics \\ Kharkiv National University \\ of Radio Electronics \\ Kharkiv, Ukraine \\ yevhenii.pashchenko@nure.ua
}

\begin{abstract}
In this work the automated module of identification of products on their visual signs is developed. The general architecture of the automated module and algorithms of work of the client module, the program in the mode of reception of the answer from the server and a server part are developed. The distributed principle of data processing and storage was used to solve the problems. The automated software module is based on the distributed control principle. Developed software for the mobile device and the necessary scripts for the server part. Testing of the developed software is performed.
\end{abstract}

Keywords-DART, Flutter, MySQL, MVC, OpenCV, SIFT, Automated Module, Intellectual Production, Customer, Recognition, Server

\section{INTRODUCTION}

The task of product identification by optical non-contact means in production is of great importance for improving product quality, reducing the percentage of waste and increasing productivity. The introduction of automated image recognition systems using modern algorithms for determining the type of components, the classification of each individual sample, regardless of its orientation in space, allows workers with lower qualifications to perform such operations. It also reduces the load on the person and reduces the number of errors when performing a particular operation.

The use of an optical system for identifying components to the warehouse makes it possible to automate such operations of the worker, which previously required only the presence of a person with a certain qualification. The integration of the automated system into the overall management system of the enterprise leads to the implementation of the requirements for the implementation of the concept of Industry 4.0 in the modern intelligent enterprise.

\section{DEVElOPMENT OF AUTOMATED MODULE ARCHITECTURE}

The distributed principle of data processing and storage was used in the development of the architecture.

In the workplace, the worker uses computer vision module, which is made using a mobile device (phone or tablet) with built-in or external camera. The resulting image is transmitted to a dedicated local or cloud server through communication. A router or Internet gateway is used as a means of communication.

The server is loaded with services for accepting images from clients, analyzing images and searching for personal features in the database. If the image has already been classified and described, the characteristics of the recognized object will be found.

The characteristics found are redirected using the appropriate tools on the server to the client that sent the recognition request. This returns the description of the object displayed on the screen of the mobile device to the operator.

Thanks to the distributed architecture and the use of a dedicated server, centralized access to information is organized for all objects that may be encountered while working at the employee's workplace. Database can be filled regardless of the main process of using the program, even in real time.

In fig. 1 shows the architecture of the automated component recognition system at the production site using the developed automated module. This figure shows a separate place of the worker (expert), who fills the database with new samples of production facilities and describes them.

\section{DeVelopment of Customer Module AlgorithM}

The algorithm of operation of the client module in the image transmission mode is shown in fig. 2. The functions of the client module.

- Get a picture of the subject with the built-in or external video camera.

- Image pre-processing.

- Connection to the server.

- Image transfer for analysis.

- Waiting for the results of the analysis.

- Display of analysis results and data about the object. 


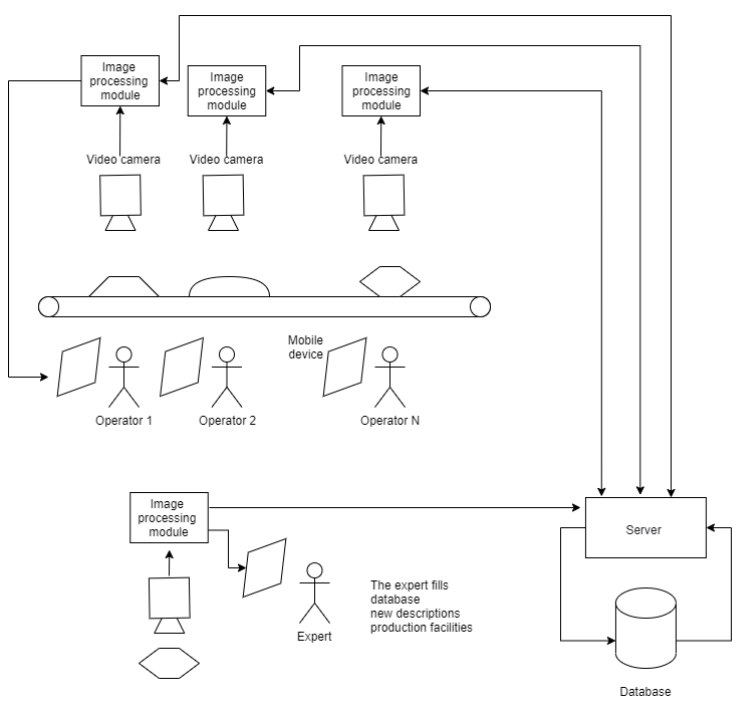

Fig. 1. Architecture of the automated system of recognition of components on a production site with use of the developed automated module

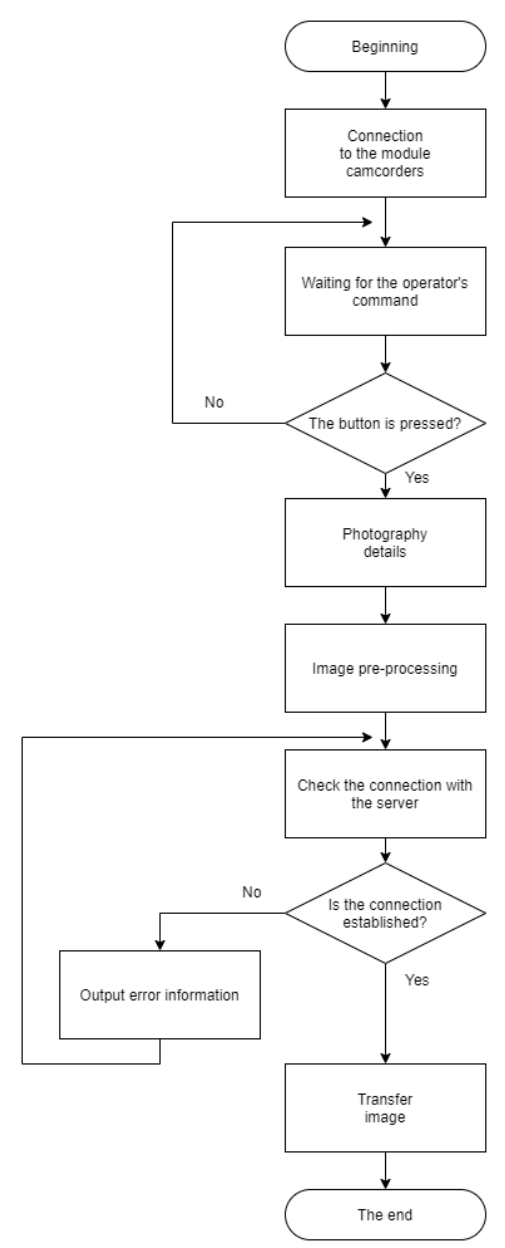

Fig. 2. Algorithm of client module operation in image transmission mode

In fig. 3 shows how the program works in the mode of receiving the image and sending it to the server. In this mode, the program is constantly configured to work with the camera and most of the time is waiting for a command from the operator. At this time, the current image of the workplace is displayed on the screen of the mobile device.

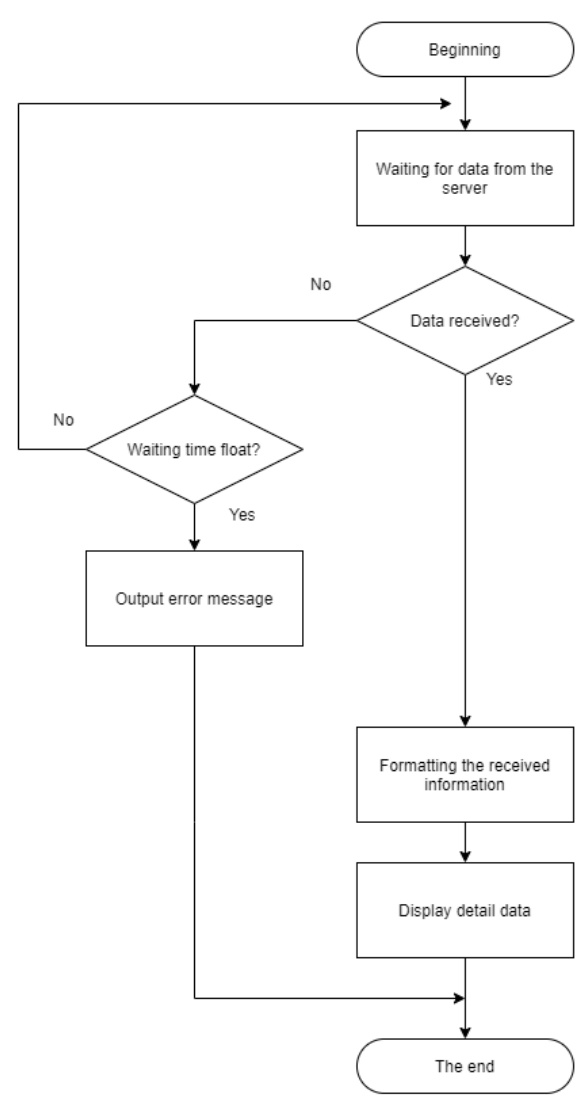

Fig. 3. Architecture of the automated system of recognition of components on a production site with use of the developed automated module

In case the operator needs to know the information about the component, he clicks on the "Recognize" button. The program takes a photo of the component that is in the workplace and pre-processes the resulting image. This removes extraneous noise and reduces the image to the specified brightness level.

After pre-processing, the connection to the server is checked. If the connection is lost, or has not yet been established, the operator displays the appropriate information window. If the connection is established, the received processed image is transmitted to the server. After that, the program goes into standby mode and receives information about the component.

In fig. 3 shows the algorithm of the program in the mode of receiving a response from the server. After sending a request to the server, the client program waits for a response. If no response is received within this time, it may indicate a connection or other problem. Thus, an error message is displayed to the operator after the set time.

If the answer is received, then before displaying it on the screen, the information is pre-formatted and only then displayed on the screen of the operator's mobile device. After these steps, the procedure of photographing and recognition is repeated.

\section{DEVElopment of THE SERVER PART Algorithm}

The functions of the server part.

- Receiving a request from the client. 
- Image verification.

- Image preparation.

- Branch on the image of key points.

- Branch of key point descriptors.

- Search in the database of similar descriptors with a certain threshold of compliance.

- Formation of the answer.

- Sending a response to the client.

The above functions are in the algorithm of the server part, which is shown in fig. 4. A descriptor is a key point identifier that distinguishes it from another mass of special points. In turn, descriptors should provide invariance of finding of correspondence between special points concerning transformations of images.

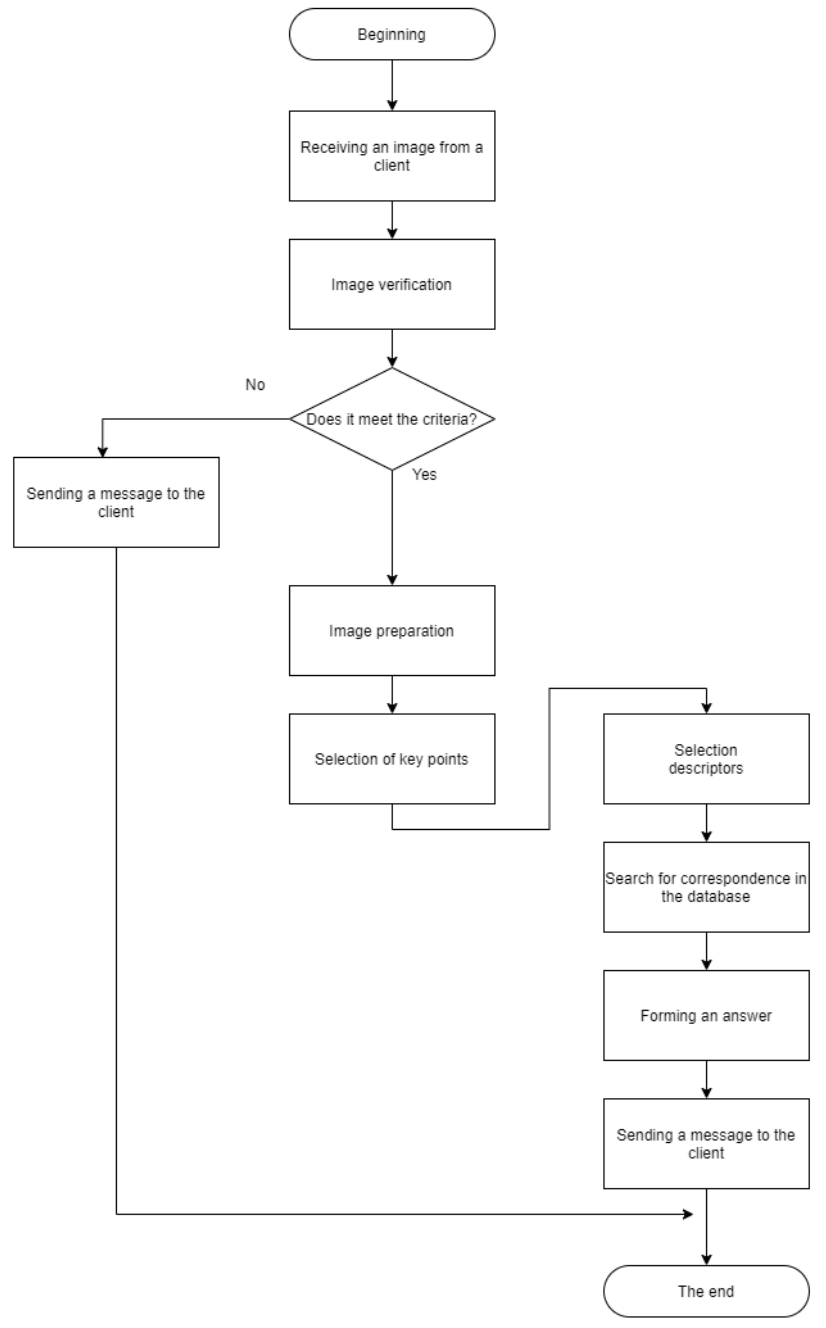

Fig. 4. Algorithm of server part operation

Preparation of the image consists in its preliminary processing on the server side. For this image is blurred by the Gaussian method with a certain radius.

Next, the image is scaled and a Gaussian pyramid is built and the difference between the obtained images is calculated. This process is shown in fig. 5 .

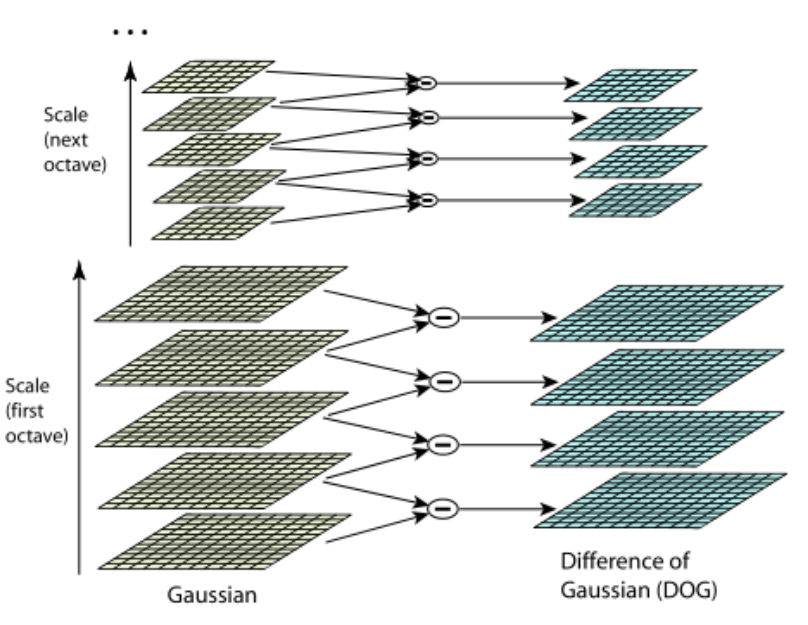

Fig. 5. Scaling and constructing Gaussian differences

This pre-treatment makes it possible to proceed to the next stage. This is the selection of special points and descriptors. The following sequence of the problem of comparison of images is used.

- Key points and their descriptors are highlighted in the images;

- By coincidence of descriptors the key points corresponding to each other are allocated;

- Based on a set of key points, an image transformation model is built, with the help of which one image can be obtained from another.

In fig. 6 shows the principle of finding descriptors in the image.

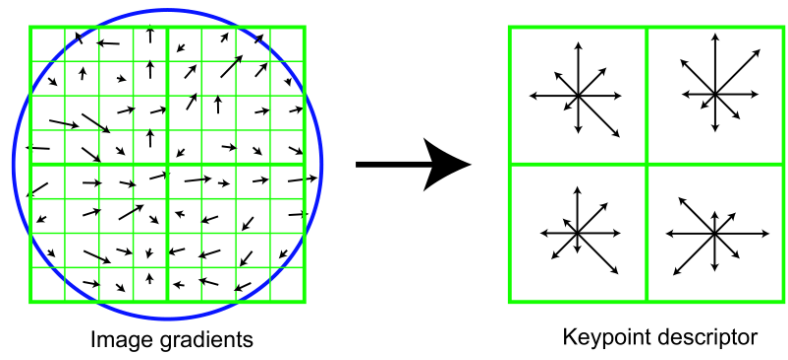

Fig. 6. Finding descriptors in the image

This figure schematically shows part of the image and obtained by the method [7] descriptor. The arrow shows the gradient of each pixel. Each descriptor gradient corresponds to three coordinates $(\mathrm{x}, \mathrm{y}, \mathrm{n}) . \mathrm{x}$ is the distance to the gradient horizontally, $\mathrm{y}$ is the distance to the gradient vertically, $\mathrm{n}$ is the distance to the gradient in the histogram. Histograms are based on the pixels of the image being processed. The value of the Gaussian nucleus is taken as a coefficient. Each occurrence of the gradient in the histogram is multiplied by all three weights of trilinear interpolation.

After processing the image and selecting the descriptors, similar descriptions are searched in the database. As a result of search the answer which is sent to the client is formed. The response may contain a description of the object, if it has been identified, or a message about the lack of information about this object, if the database does not match. 


\section{Software DeVelopment AND Testing}

Developed for a mobile device, the program has a graphical interface with the ability to view the database, download new components to the database and recognize components in real time with the presentation of information from the database.

Each component has a name, a short description, a photo from different angles, the cell number in the warehouse. The country of manufacture and the city where the production is located are also indicated. The user has the ability to add the name of the component, country of manufacture, city, specify the type of part and the city of storage in the warehouse. The user can also add the necessary additional images of the component. These can be fragments of the drawing, or additional views (fig.7).

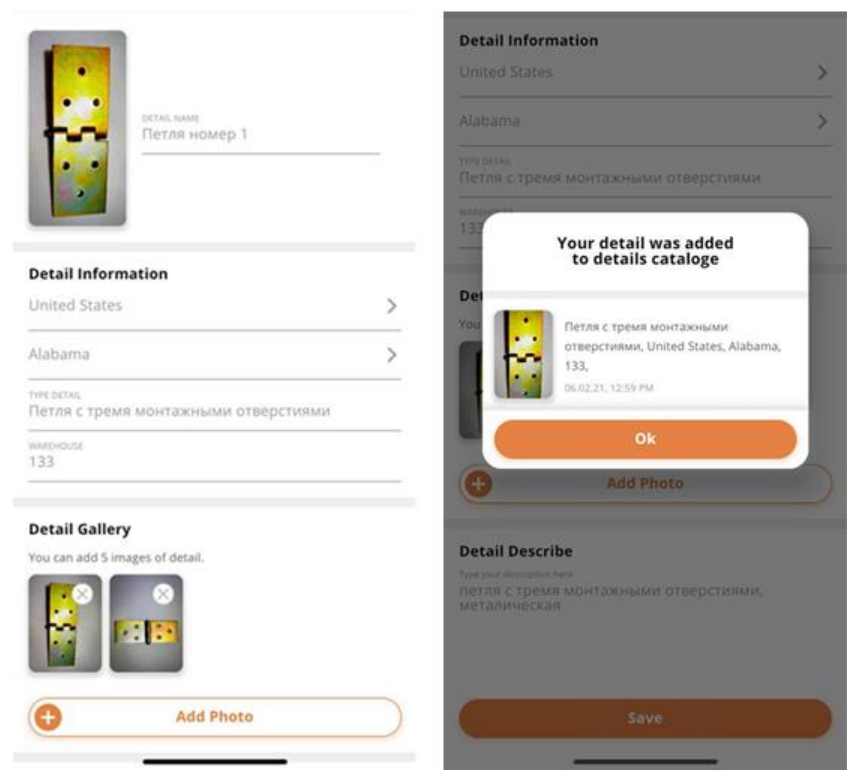

Fig. 7. Example of two software screens

To test the developed software, a database of components was created and a list of all items in the database was obtained using the appropriate window.

Next, the recognition algorithm was tested. The recognition accuracy is affected by the coefficient of the distance of special points, which is specified in the code by the server. Changing the specified coefficient, a number of test tests were performed.

In fig. 8 shows a graph of the dynamics of the number of errors from the distance coefficient of special points. As a result of the results analysis of recognition algorithm testing it is possible to define optimum value of factor of distance of special points making 0,55 .

The recognition results are also affected by the color scheme of the image. So images in the format "IMREAD_REDUCED_COLOR_4" have more cases of correct recognition.

\section{CONCLUSION}

In this work the automated module of identification of products on their visual signs is developed. The general architecture of the automated module and algorithms of work of the client module, the program in the mode of reception of the answer from the server and a server part are developed.

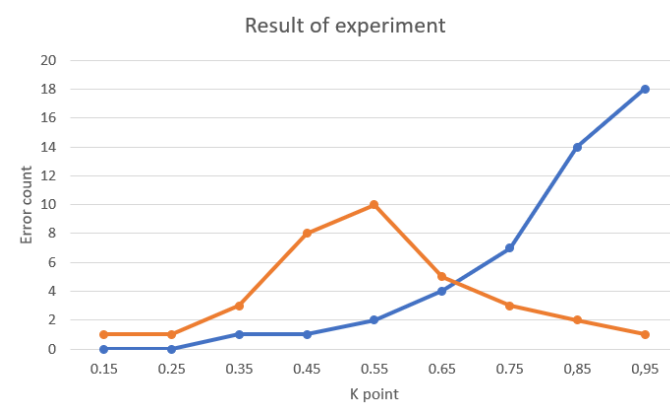

Fig. 8. The results of testing the recognition algorithm

The distributed principle of data processing and storage was used to solve the problems. The automated software module is based on the distributed control principle. The server uses the following technologies and programming languages: Laravel framework, PHP programming language, MySQL database, Open CV. The Flutter framework and the Dart programming language are used to write the program.

Developed software for the mobile device and the necessary scripts for the server part. Testing of the developed software is performed. As a result of the analysis of the results of testing the recognition algorithm, it is possible to determine the optimal value of the distance coefficient of special points, which is 0.55 . It was also found that the recognition results are influenced by the color scheme of the image. So images in the format "IMREAD_REDUCED_COLOR_4" have more cases of correct recognition.

\section{REFERENCES}

[1] D.G. Lowe, "Object recognition from local scale-invariant features," Proceedings of the International Conference on Computer Vision, vol. 2, 1999, pp. 1150-1157.

[2] D.G. Lowe, "Distinctive Image Features from Scale-Invariant Keypoints," International Journal of Computer Vision, vol. 60, issue 2, 2004, pp. 91-110.

[3] T. Serre, M. Kouh, C. Cadieu, U. Knoblich, and etc., "A Theory of Object Recognition: Computations and Circuits in the Feedforward Path of the Ventral Stream in Primate Visual Cortex," Computer Science and Artificial Intelligence Laboratory Technical Report, December 19, 2005.

[4] B. Herbert, A. Ess, T. Tuytelaars, L. Van Gool, "SURF: Speeded Up Robust Features," Computer Vision and Image Understanding (CVIU), vol. 110, issue. 3, 2008, pp. 346-359.

[5] G. Takacs, V. Chandrasekhar, S. Tsai, D. Chen, R. Grzeszczuk and B. Girod, "Unified Real-Time Tracking and Recognition with RotationInvariant Fast Features," 2010 IEEE Computer Society Conference on Computer Vision and Pattern Recognition, 2010, pp. 934-941, doi: 10.1109/CVPR.2010.5540116.

[6] Calonder M., Lepetit V., Strecha C., Fua P. (2010) BRIEF: Binary Robust Independent Elementary Features. In: Daniilidis K., Maragos P., Paragios N. (eds) Computer Vision - ECCV 2010. ECCV 2010. Lecture Notes in Computer Science, vol 6314. Springer, Berlin, Heidelberg. https://doi.org/10.1007/978-3-642-15561-1_56.

[7] SIFT descriptor construction and image matching task. [Online]. Available: https://habr.com/ru/post/106302/ [Accessed: 5- Jun- 2021] 